\title{
An elementary unified approach to prove some identities involving Fibonacci and Lucas numbers
}

\author{
Moussa Benoumhani \\ Department of Mathematics, University of Msila \\ Msila, Algeria \\ e-mail: moussa.benoumhani@univ-msila.dz
}

Received: 3 November 2020 Revised: 15 November $2021 \quad$ Accepted: 3 December 2021

\begin{abstract}
Using the explicit formulas of the generating polynomials of Fibonacci and Lucas, we prove some new identities involving Fibonacci and Lucas numbers. As an application of these identities, we show how some Diophantine equations have infinitely many solutions. To illustrate the powerful of this elementary method, we give proofs of many known formulas.
\end{abstract}

Keywords: Fibonacci number, Lucas number, Polynomials, Diophantine equation.

2020 Mathematics Subject Classification: 11B39, 11 B65.

\section{Introduction}

The huge number of identities and relations between Fibonacci (and Lucas) numbers may fill many books. These relations are deduced via many different methods, certainly almost all of them are elementary. Perhaps, the most powerful (non elementary) tool is the hypergeometric functions method. This method gives many beautiful identities (finite and infinite) and is used just in a sporadic papers, see for instance $[3,5]$ and the references therein.

There is no (known) general unified approach to derive identities or relations between the Fibonacci (and Lucas) numbers. May be the most general formula, based on the explicit formula of the Fibonacci polynomial and its anti derivative is due to Seiffert [13]. Nevertheless, we remarked that considering the Fibonacci generating polynomial and its relatives (its reciprocal, derivative, anti derivative), with appropriate substitutions supply easy (and different) proofs of 
many identities, and (may) lead to "new" identities too. It seems that Fibonacci polynomials are not yet well exploited. Finally, for the sentence "new identity", we cite Dilcher [5]: "Statements that a certain identity is new should be taken with the necessary caution". Indeed, the vastness of the literature in the subject make it hard to check it exhaustively.

The aim of this paper is to prove many identities and results concerning Fibonacci and Lucas numbers, using only Fibonacci and Lucas polynomials, their reciprocal polynomials, and sometimes their derivatives and anti derivatives. In the next section, we recall the polynomials we will use in the sequel, as well as some classical basic relations convenient for specializations. In Section 3 we prove some new identities. In Section 4, we use these identities to solve many Diophantine equations. The fifth section is devoted essentially to the proofs of the results of $[7,9,14]$. These results are chosen because they are relatively recent and are of the same type. In Section 6, using the derivatives of Fibonacci polynomials, we prove other known results due to Melham [12]. In the last section, we discuss further questions and state some conjectures.

\section{Preliminaries}

For every $n \geq 2$, Fibonacci polynomials sequence is defined by

$$
\phi_{n}(x)=\phi_{n-1}(x)+x \phi_{n-2}(x), \phi_{0}(x)=0, \phi_{1}(x)=1 .
$$

The explicit formula of $\phi_{n}(x)$ is given by

$$
\begin{aligned}
\phi_{n}(x) & =\sum_{k=0}^{\left\lfloor\frac{n-1}{2}\right\rfloor}\left(\begin{array}{c}
n-k-1 \\
k
\end{array}\right) x^{k} \\
& =\frac{1}{\sqrt{4 x+1}}\left(\left(\frac{1+\sqrt{4 x+1}}{2}\right)^{n}-\left(\frac{1-\sqrt{4 x+1}}{2}\right)^{n}\right) .
\end{aligned}
$$

We will use $\phi_{2 n}(x)$

$$
\begin{aligned}
\phi_{2 n}(x) & =\sum_{k=0}^{n-1}\left(\begin{array}{c}
2 n-k-1 \\
k
\end{array}\right) x^{k} \\
& =\frac{1}{\sqrt{4 x+1}}\left(\left(\frac{1+\sqrt{4 x+1}}{2}\right)^{2 n}-\left(\frac{1-\sqrt{4 x+1}}{2}\right)^{2 n}\right) .
\end{aligned}
$$

Recall that if $P_{n}(x)=\sum_{k=0}^{n} a_{k} x^{k}$ is a polynomial, its reciprocal $P_{n}^{r c}(x)$ is defined by

$$
P_{n}^{r c}(x)=x^{n} P_{n}\left(\frac{1}{x}\right)=\sum_{k=0}^{n} a_{n-k} x^{k} .
$$

So,

$$
F_{2 n}(x)=\phi_{2 n}^{r c}(x)=\sum_{k=0}^{n-1}\left(\begin{array}{c}
n+k \\
2 k+1
\end{array}\right) x^{k}
$$

and

$$
F_{2 n+1}(x)=\sum_{k=0}^{n}\left(\begin{array}{c}
n+k \\
2 k
\end{array}\right) x^{k}
$$


Then

$$
F_{2 n}(x)=\frac{1}{\sqrt{x(x+4)}}\left(\left(\frac{\sqrt{x}+\sqrt{4+x}}{2}\right)^{2 n}-\left(\frac{\sqrt{x}-\sqrt{4+x}}{2}\right)^{2 n}\right),
$$

and

$$
F_{2 n+1}(x)=\frac{1}{\sqrt{x+4}}\left(\left(\frac{\sqrt{x}+\sqrt{4+x}}{2}\right)^{2 n+1}-\left(\frac{\sqrt{x}-\sqrt{4+x}}{2}\right)^{2 n+1}\right) .
$$

Recall the very known explicit formulas, respectively for Fibonacci and Lucas numbers:

$$
\begin{aligned}
F_{n} & =\frac{1}{\sqrt{5}}\left(\left(\frac{1+\sqrt{5}}{2}\right)^{n}-\left(\frac{1-\sqrt{5}}{2}\right)^{n}\right) \\
& =\frac{1}{\sqrt{5}}\left(\alpha^{n}-\beta^{n}\right),
\end{aligned}
$$

with $F_{0}=0, F_{1}=1$. For Lucas numbers $\left(L_{n}\right)$, we have $L_{0}=2, L_{1}=1$, and for $n \geq 2$

$$
L_{n}=\alpha^{n}+\beta^{n} .
$$

The next relation is also very famous

$$
\begin{aligned}
& 5 F_{l}^{2}+4(-1)^{l}=L_{l}^{2} \\
& 5 F_{l}^{2}(-1)^{l}+4=(-1)^{l} L_{l}^{2} .
\end{aligned}
$$

The antiderivative of $F_{n}(x)$ will be useful: for $n \geq 1$, we have

$$
\begin{aligned}
G_{2 n}(x) & =\int_{0}^{x} \sum_{k=0}^{n-1}\left(\begin{array}{c}
n+k \\
2 k+1
\end{array}\right) t^{k} d t=\sum_{k=0}^{n-1}\left(\begin{array}{c}
n+k \\
2 k+1
\end{array}\right) \frac{x^{k+1}}{k+1} \\
& =\frac{1}{n}\left(\left(\frac{\sqrt{x}+\sqrt{4+x}}{2}\right)^{2 n}+\left(\frac{\sqrt{x}-\sqrt{4+x}}{2}\right)^{2 n}\right)-\frac{2}{n},
\end{aligned}
$$

and

$$
\begin{aligned}
H_{2 n+1}(x) & =\int_{0}^{x} \sum_{k=0}^{n}\left(\begin{array}{c}
n+k \\
2 k
\end{array}\right) t^{2 k} d t=\sum_{k=0}^{n}\left(\begin{array}{c}
n+k \\
2 k
\end{array}\right) \frac{x^{2 k+1}}{2 k+1} \\
& =\frac{1}{2 n+1}\left(\left(\frac{x+\sqrt{4+x^{2}}}{2}\right)^{2 n+1}-\left(\frac{x-\sqrt{4+x^{2}}}{2}\right)^{2 n+1}\right) .
\end{aligned}
$$

The last polynomials we need are

$$
\begin{gathered}
\sum_{k=0}^{\left\lfloor\frac{n-1}{2}\right\rfloor}\left(\begin{array}{c}
n \\
2 k+1
\end{array}\right) x^{2 k}=\frac{1}{2 x}\left((1+x)^{n}-(1-x)^{n}\right) \\
\sum_{k=0}^{\left\lfloor\frac{n-1}{2}\right\rfloor}\left(\begin{array}{c}
n \\
2 k
\end{array}\right) x^{2 k}=\frac{1}{2}\left((1+x)^{n}+(1-x)^{n}\right) .
\end{gathered}
$$




\section{Some new identities}

In this section, we prove some identities involving Fibonacci and Lucas numbers. The proof as remarked before is based on the explicit formula of Fibonacci generating polynomial. Some of these identities seem to be new.

Theorem 3.1. We have for all $n \geq 1$ and $l \geq 1$

$$
\begin{aligned}
n \sum_{k=0}^{n-1}\left(\begin{array}{c}
n+k \\
2 k+1
\end{array}\right) \frac{(-1)^{l(n+k+1)}\left(5 F_{l}^{2}\right)^{k}}{k+1} & =\left(\frac{F_{n l}}{F_{l}}\right)^{2} \\
n \sum_{k=0}^{n-1}\left(\begin{array}{c}
n+k \\
2 k+1
\end{array}\right) \frac{(-1)^{(l+1)(n+k+1)}\left(L_{l}^{2}\right)^{k+1}}{k+1} & =L_{2 n l}-2(-1)^{n(l+1)} \\
n \sum_{k=0}^{n-1}\left(\begin{array}{c}
n+k \\
2 k+1
\end{array}\right) \frac{(-1)^{(n+k+1)}}{k+1} & =2 \cos \left(\frac{n \pi}{3}\right)-2 \\
(2 n+1) \sum_{k=0}^{n}\left(\begin{array}{c}
n+k \\
2 k
\end{array}\right) \frac{(-1)^{n+k}}{2 k+1} & =2 \cos \frac{(2 n+1) \pi}{6} .
\end{aligned}
$$

Proof. Replace $x$ by $x^{2}$ in (6) yields

$$
\sum_{k=0}^{n-1}\left(\begin{array}{c}
n+k \\
2 k+1
\end{array}\right) \frac{(x)^{2 k+2}}{k+1}+\frac{2}{n}=\frac{1}{n}\left(\left(\frac{x+\sqrt{4+x^{2}}}{2}\right)^{2 n}+\left(\frac{x-\sqrt{4+x^{2}}}{2}\right)^{2 n}\right) .
$$

Let $x=i^{l} \sqrt{5} F_{l}$ in the previous relation:

$$
\begin{aligned}
& \sum_{k=0}^{n-1}\left(\begin{array}{c}
n+k \\
2 k+1
\end{array}\right) \frac{\left(i^{l} \sqrt{5} F_{l}\right)^{2 k+2}}{k+1}+\frac{2}{n}=\frac{1}{n}\left(\left(\frac{i^{l} \sqrt{5} F_{l}+i^{l} L_{l}}{2}\right)^{2 n}+\left(\frac{i^{l} \sqrt{5} F_{l}-i^{l} L_{l}}{2}\right)^{2 n}\right) \\
& \sum_{k=0}^{n-1}\left(\begin{array}{c}
n+k \\
2 k+1
\end{array}\right) \frac{(-1)^{l(k+1)}\left(5 F_{l}^{2}\right)^{k+1}}{k+1}=\frac{(-1)^{n l}}{n}\left(\alpha^{2 n l}+\beta^{2 n l}\right)-\frac{2}{n}=\frac{(-1)^{n l}}{n}\left(L_{2 n l}-2(-1)^{n l}\right) \\
& \sum_{k=0}^{n-1}\left(\begin{array}{c}
n+k \\
2 k+1
\end{array}\right) \frac{(-1)^{l(k+1)}\left(5 F_{l}^{2}\right)^{k+1}}{k+1}=\frac{(-1)^{n l}}{n}\left(\alpha^{2 n l}+\beta^{2 n l}-2(-1)^{n l}\right)=\frac{(-1)^{n l}}{n}\left(\alpha^{n l}-\beta^{n l}\right)^{2},
\end{aligned}
$$

which is:

$$
\left(\frac{F_{n l}}{F_{l}}\right)^{2}=n \sum_{k=0}^{n-1}\left(\begin{array}{c}
n+k \\
2 k+1
\end{array}\right) \frac{(-1)^{l(n+k+1)}\left(5 F_{l}^{2}\right)^{k}}{k+1} .
$$

For the second identity, again let $x=i^{l+1} L_{l}$ in (6), we get:

$$
\begin{aligned}
G_{2 n}\left(i^{l+1} L_{l}\right) & =\sum_{k=0}^{n-1}\left(\begin{array}{c}
n+k \\
2 k+1
\end{array}\right) \frac{\left(i^{l+1} L_{l}\right)^{2 k+2}}{k+1}+\frac{2}{n} \\
& =\frac{1}{n}\left(\left(\frac{i^{l+1} L_{l}+i^{l+1} \sqrt{5} F_{l}}{2}\right)^{2 n}+\left(\frac{i^{l+1} L_{l}-i^{l+1} \sqrt{5} F_{l}}{2}\right)^{2 n}\right) .
\end{aligned}
$$


Note that

$$
\left(\frac{i^{l+1} L_{l}+i^{l+1} \sqrt{5} F_{l}}{2}\right)^{2 n}=(-1)^{n(l+1)} \alpha^{2 n l}
$$

and

Rearrange to obtain

$$
\left(\frac{i^{l+1} L_{l}-i^{l+1} \sqrt{5} F_{l}}{2}\right)^{2 n}=(-1)^{n(l+1)} \beta^{2 n l} .
$$

$$
\begin{aligned}
n \sum_{k=0}^{n-1}\left(\begin{array}{c}
n+k \\
2 k+1
\end{array}\right) \frac{(-1)^{(l+1)(n+k+1)}\left(L_{l}^{2}\right)^{k+1}}{k+1} & =\left(\alpha^{2 n l}+\beta^{2 n l}\right)-(-1)^{n(l+1)} 2 \\
& =L_{2 n l}-(-1)^{n(l+1)} 2,
\end{aligned}
$$

which is the desired formula. The last identities are obtained by setting $x=i$ in (6) and (7).

For $l=1,2$, we obtain

$$
\begin{aligned}
F_{n}^{2} & =n \sum_{k=0}^{n-1}\left(\begin{array}{c}
n+k \\
2 k+1
\end{array}\right) \frac{(-1)^{(n+k+1)}(5)^{k}}{k+1} \\
F_{2 n}^{2} & =n \sum_{k=0}^{n-1}\left(\begin{array}{c}
n+k \\
2 k+1
\end{array}\right) \frac{5^{k}}{k+1} .
\end{aligned}
$$

Those formulas appear, as particular cases in [13], but it does not seem possible to extract $F_{n l}^{2}$ from Seifert general formula for every $l \geq 1$.

Corollary 3.1. For positive integers $n, l \geq 1$, we have

$$
\begin{gathered}
\left(\frac{F_{n l}}{L_{l}}\right)^{2}=\frac{2 n}{5} \sum_{k=0}^{2 n-1}\left(\begin{array}{c}
2 n+k \\
2 k+1
\end{array}\right) \frac{(-1)^{(l+1)(k+1)}\left(L_{l}^{2}\right)^{k}}{k+1}, \\
\left(\frac{L_{(2 n+1) l}}{L_{l}}\right)^{2}=(2 n+1) \sum_{k=0}^{2 n}\left(\begin{array}{c}
2 n+k+1 \\
2 k+1
\end{array}\right) \frac{(-1)^{k(l+1)}\left(L_{l}^{2}\right)^{k}}{k+1} .
\end{gathered}
$$

Proof. From the second relation in the previous theorem

$$
L_{2 n l}-2(-1)^{n(l+1)}=\left(\alpha^{n l}-\beta^{n l}\right)^{2}+2(-1)^{n l}-2(-1)^{n(l+1)} .
$$

For $n$ even, the last relation becomes

$$
L_{4 n l}-2(-1)^{2 n(l+1)}=\left(\alpha^{2 n l}-\beta^{2 n l}\right)^{2},
$$

dividing by 5 , yields the first relation of the corollary. For the second one, we have

$$
\begin{aligned}
L_{2 n l}-2(-1)^{n(l+1)} & =\left(\alpha^{n l}+\beta^{n l}\right)^{2}-2(-1)^{n l}-2(-1)^{n(l+1)} \\
& =L_{n l}^{2}-2(-1)^{n l}\left(1+(-1)^{n}\right) .
\end{aligned}
$$

For $n$ odd, we obtain

$$
\begin{aligned}
L_{2(2 n+1) l}-2(-1)^{(2 n+1)(l+1)} & =\left(\alpha^{(2 n+1) l}+\beta^{(2 n+1) l}\right)^{2} \\
& =L_{(2 n+1) l}^{2},
\end{aligned}
$$

which is the wanted formula. 
Corollary 3.2. For every integer $n \geq 1$

$$
\begin{aligned}
(2 n+1) & \sum_{k=0}^{2 n}\left(\begin{array}{c}
2 n+k+1 \\
2 k+1
\end{array}\right) \frac{(-4)^{k}}{k+1}=1 \\
& \frac{2 n}{5} \sum_{k=0}^{2 n-1}\left(\begin{array}{c}
2 n+k \\
2 k+1
\end{array}\right) \frac{(-4)^{k}}{k+1}=0 .
\end{aligned}
$$

Proof. Let $l=0$, in the previous relations.

It is possible to find other identities, using the polynomials

$$
\begin{gathered}
\sum_{k=0}^{\left\lfloor\frac{n-1}{2}\right\rfloor}\left(\begin{array}{c}
n \\
2 k+1
\end{array}\right) x^{2 k}=\frac{1}{2 x}\left((1+x)^{n}-(1-x)^{n}\right) \\
\sum_{k=0}^{\left\lfloor\frac{n-1}{2}\right\rfloor}\left(\begin{array}{c}
n \\
2 k
\end{array}\right) x^{2 k}=\frac{1}{2}\left((1+x)^{n}+(1-x)^{n}\right) .
\end{gathered}
$$

For example Catalan's well known identity

$$
F_{n}=2^{1-n} \sum_{k=0}^{\left\lfloor\frac{n-1}{2}\right\rfloor}\left(\begin{array}{c}
n \\
2 k+1
\end{array}\right) 5^{k}
$$

is obtained by setting $x=\sqrt{5}$ in (12). In fact Catalan's identity is a particular case of the

Theorem 3.2. For every $l \geq 1, n \geq 1$, we have

$$
\begin{aligned}
& F_{n l}=2^{1-n} F_{l} L_{l}^{n-1} \sum_{k=0}^{\left\lfloor\frac{n-1}{2}\right\rfloor}\left(\begin{array}{c}
n \\
2 k+1
\end{array}\right)\left(\frac{5 F_{l}^{2}}{L_{l}^{2}}\right)^{k} \\
& L_{n l}=2^{1-n} L_{l}^{n} \sum_{k=0}^{\left\lfloor\frac{n-1}{2}\right\rfloor}\left(\begin{array}{c}
n \\
2 k
\end{array}\right)\left(\frac{5 F_{l}^{2}}{L_{l}^{2}}\right)^{k} .
\end{aligned}
$$

Proof. Put $x=\frac{\sqrt{5} F_{l}}{L_{l}}$, in the previous polynomials.

For $l=1, F_{n}=2^{1-n} \sum_{k=0}^{\left\lfloor\frac{n-1}{2}\right\rfloor}\left(\begin{array}{c}n \\ 2 k+1\end{array}\right) 5^{k}$ is Catalan identity. For $l=2$,

$$
F_{2 n}=\left(\frac{3}{2}\right)^{n-1} \sum_{k=0}^{\left\lfloor\frac{n-1}{2}\right\rfloor}\left(\begin{array}{c}
n \\
2 k+1
\end{array}\right)\left(\frac{5}{9}\right)^{k}
$$

this is relation (5.9) in [5]. For $l=3$, we have $F_{3 n}=2^{n} \sum_{k=0}^{\left\lfloor\frac{n-1}{2}\right\rfloor}\left(\begin{array}{c}n \\ 2 k+1\end{array}\right)\left(\frac{5}{4}\right)^{k}, \ldots$ 
Theorem 3.3. For every integers $n, l \geq 1$, we have

$$
F_{n l}=F_{l} \sum_{k=0}^{\left\lfloor\frac{n-1}{2}\right\rfloor}\left(\begin{array}{c}
n-k-1 \\
k
\end{array}\right)(-1)^{k(l+1)} L_{l}^{n-2 k-1},
$$

and

$$
\sum_{k=0}^{\left\lfloor\frac{n-1}{2}\right\rfloor}\left(\begin{array}{c}
n-k-1 \\
k
\end{array}\right)(-1)^{k l}\left(\frac{1}{5 F_{l}^{2}}\right)^{k}=\left\{\begin{array}{c}
\frac{F_{n l}}{5^{\frac{n-2}{2}} F_{l}^{n-1} L_{l}}, n=2 m \\
\frac{L_{n l}}{\left(\sqrt{5} F_{l}\right)^{n-1} L_{l}}, n=2 m+1 .
\end{array}\right.
$$

Proof. Let $x=\frac{(-1)^{l+1}}{L_{l}^{2}}$ in the first polynomial, we obtain the first formula. This is (6) in [8]. For the second identity, let $x=\frac{(-1)^{l}}{5 F_{l}^{2}}$; we get

$$
\begin{aligned}
\sum_{k=0}^{\left\lfloor\frac{n-1}{2}\right\rfloor}\left(\begin{array}{c}
n-k-1 \\
k
\end{array}\right)(-1)^{k l}\left(\frac{1}{5 F_{l}^{2}}\right)^{k} & =\frac{\sqrt{5} F_{l}}{L_{l}}\left(\left(\frac{\alpha^{l}}{\sqrt{5} F_{l}}\right)^{n}-\left(\frac{-\beta^{l}}{\sqrt{5} F_{l}}\right)^{n}\right) \\
& =\frac{1}{L_{l}\left(\sqrt{5} F_{l}\right)^{n-1}}\left(\alpha^{n l}-(-1)^{n} \beta^{n l}\right) .
\end{aligned}
$$

So, we obtain

$$
\sum_{k=0}^{\left\lfloor\frac{n-1}{2}\right\rfloor}\left(\begin{array}{c}
n-k-1 \\
k
\end{array}\right)(-1)^{k l}\left(\frac{1}{5 F_{l}^{2}}\right)^{k}=\left\{\begin{array}{c}
\frac{F_{n l}}{5^{\frac{n-2}{2}} F_{l}^{n-1} L_{l}}, n=2 m \\
\frac{L_{n l}}{\left(\sqrt{5} F_{l}\right)^{n-1} L_{l}}, n=2 m+1 .
\end{array}\right.
$$

We can also write these formulas separately as follows:

$$
\begin{gathered}
F_{2 m l}=\frac{L_{l}}{5 F_{l}} \sum_{k=0}^{m-1}(-1)^{k l}\left(\begin{array}{c}
2 m-k-1 \\
k
\end{array}\right)\left(5 F_{l}^{2}\right)^{m-k} \\
L_{(2 m+1) l}=L_{l} \sum_{k=0}^{m}(-1)^{k l}\left(\begin{array}{c}
2 m-k \\
k
\end{array}\right)\left(5 F_{l}^{2}\right)^{m-k} .
\end{gathered}
$$

Those do not appear in [8].

Another less well known identity involving (1) is

$$
n=\sum_{k=0}^{\left\lfloor\frac{n-1}{2}\right\rfloor}(-1)^{k}\left(\begin{array}{c}
n-k-1 \\
k
\end{array}\right) 2^{n-2 k-1},
$$

this is due to Lucas, see [8]. It may be obtained as a limit, in fact since $F_{n}(x)$ is a polynomial then it is defined for every $x \in \mathbb{R}$, and then

$$
F_{n}\left(-\frac{1}{4}\right)=\lim _{x \longrightarrow-\frac{1}{4}} \frac{\left(\frac{1+\sqrt{4 x+1}}{2}\right)^{n}-\left(\frac{1-\sqrt{4 x+1}}{2}\right)^{n}}{\sqrt{4 x+1}}=\frac{n}{2^{n-1}},
$$

which is the wanted identity. By the same we deduce the following corollary. 
Corollary 3.3. For $n \geq 1$

$$
n=\sum_{k=0}^{n-1}(-1)^{n+k+1}\left(\begin{array}{c}
n+k \\
2 k+1
\end{array}\right) 4^{k} .
$$

Proof. This may be deduced by replacing $n$ by $2 n$ in the previous proposition, or as a limit:

$$
\begin{aligned}
F(-4) & =\sum_{k=0}^{n-1}(-1)^{k}\left(\begin{array}{c}
n+k \\
2 k+1
\end{array}\right) 4^{k} \\
& =\lim _{x \longrightarrow-4} \frac{\left(\frac{\sqrt{x}+\sqrt{x+4}}{2}\right)^{n}-\left(\frac{\sqrt{x}-\sqrt{x+4}}{2}\right)^{n}}{\sqrt{x(x+4)}}=(-1)^{n+1} n .
\end{aligned}
$$

This is the analogous of (13).

\section{Application to some Diophantine equations}

It is possible to use the previous results to see that some surfaces contain many lattice points, which are Fibonacci and/or Lucas numbers. Also, we will see that the solutions of some Diophantine equations, are sequences of these numbers

Theorem 4.1. The following surfaces contain infinitely many lattice points:

$$
z^{2}=5 x^{2} \pm 4 y^{2}
$$

Proof. Of course, all of the solutions of these equations may be easily determined. But, it is nice to know that some are Fibonacci and Lucas numbers. In fact, letting $n=1$, in the first relation of Corollary 2, yields:

$$
5 F_{2 l}^{2}=4(-1)^{l+1} L_{l}^{2}+\left(L_{l}^{2}\right)^{2} .
$$

This may be written, according to $l$ odd or even, as follows:

$$
\begin{aligned}
\left(L_{2 l}^{2}\right)^{2} & =5 F_{4 l}^{2}+4 L_{2 l}^{2} \\
\left(L_{2 l+1}^{2}\right)^{2} & =5 F_{4 l+2}^{2}-4 L_{2 l+1}^{2} .
\end{aligned}
$$

This means that $(z, y, x)=\left(L_{2 l}, F_{4 l}, L_{2 l}\right)$ and $\left(L_{2 l+1}, F_{4 l+2}, L_{2 l+1}\right)$ are families of integer solutions of these equations.

Theorem 4.2. The following equations have infinitely many solutions which are Fibonacci and Lucas numbers:

1) $5 y^{2}=x^{3} \pm 8 x^{2}+20 x \pm 16$

2) $z^{2}=x^{4} \pm 6 y^{2}+9$

Proof. Note that the first equations are singular elliptic curves:

$$
5 y^{2}=x^{3} \pm 8 x^{2}+20 x \pm 16=(x \pm 4)(x \pm 2)^{2} .
$$

So, we can find all the solutions. But it is nice to see that among these solutions, infinitely are Fibonacci and Lucas numbers. Let $n=2$ in relation 1 of Corollary 2. We obtain 


$$
\begin{aligned}
5\left(\frac{F_{2 l}}{L_{l}}\right)^{2} & =4 \sum_{k=0}^{3}\left(\begin{array}{c}
4+k \\
2 k+1
\end{array}\right) \frac{(-1)^{(l+1)(k+1)}\left(L_{l}^{2}\right)^{k}}{k+1} . \\
& =L_{l}^{3}+8(-1)^{l+1}\left(L_{l}^{2}\right)^{2}+20\left(L_{l}^{2}\right)+(-1)^{l+1} 16 .
\end{aligned}
$$

So, the pairs $\left(\frac{F_{2 l}}{L_{l}}, L_{l}^{2}\right), l \geq 1$, is a set of solutions for the first equations. For the second ones, let $n=1$ in the second relation of Corollary 2, to get:

$$
\begin{aligned}
\left(\frac{L_{3 l}}{L_{l}}\right)^{2} & =3 \sum_{k=0}^{2}\left(\begin{array}{c}
3+k \\
2 k+1
\end{array}\right) \frac{(-1)^{k(l+1)}\left(L_{l}^{2}\right)^{k}}{k+1} \\
& =9+6(-1)^{l+1} L_{l}^{2}+L_{l}^{4} .
\end{aligned}
$$

This means that $\left(\frac{L_{6 l+3}}{L_{2 l+1}}, L_{2 l+1}, L_{2 l+1}\right)$ and $\left(\frac{L_{6 l}}{L_{2 l}}, L_{2 l}, L_{2 l}\right), l \geq 0$ are families of solutions for the equations $t^{2}=x^{4} \pm 6 y^{2}+9$.

Remark 4.1. Using the identities proved in the previous sections, it is possible to find solutions to other Diophantine equations, in the same vein as in the $[1,10]$.

\section{Some known identities involving Fibonacci numbers}

In this section, we prove some identities involving Fibonacci and Lucas numbers. All of them are known and due to Jennings [9], Filipponi [7] and Swamy [14]. Curiously, all of these identities are easily derived via the generating polynomial $F_{n}(x)$ and an almost trivial substitutions. Let us illustrate this fact by the following proposition:

Proposition 5.1. For every $n \geq 1$, we have

$$
\begin{aligned}
& F_{4 n}=3(-1)^{n-1} \sum_{k=0}^{n-1}\left(\begin{array}{c}
n+k \\
2 k+1
\end{array}\right)(-9)^{k} \\
& F_{2 n}=(-1)^{n-1} \sum_{k=0}^{n-1}(-1)^{k}\left(\begin{array}{c}
n+k \\
2 k+1
\end{array}\right) 5^{k} \\
& F_{4 n}=3 \sum_{k=0}^{n-1}\left(\begin{array}{c}
n+k \\
2 k+1
\end{array}\right) 5^{k} .
\end{aligned}
$$

Proof. The first and the last identity are obtained by setting, repectively, $x=-9$, and then $x=5$ in (2) and remembering that $\left(\frac{1 \pm \sqrt{5}}{2}\right)^{2}=\frac{3 \pm \sqrt{5}}{2}$. To obtain the second put $x=-5$ in (2).

These are known, and may be found in [5]. In fact those are particular cases of the following general ones: 
Theorem 5.1. (Jennings [9]) For every integers $n, l \geq 0$

$$
\begin{aligned}
F_{2 n l} & =F_{l} \sum_{k=0}^{n-1}(-1)^{(l+1)(n+k+1)}\left(\begin{array}{c}
n+k \\
2 k+1
\end{array}\right) L_{l}^{2 k+1} \\
F_{(2 n+1) l} & =F_{l} \sum_{k=0}^{n}(-1)^{(l+1)(n+k)}\left(\begin{array}{c}
n+k \\
2 k
\end{array}\right) L_{l}^{2 k} . \\
F_{(2 n+1) l} & =F_{l} \sum_{k=0}^{n}(-1)^{l(n+k)} \frac{2 n+1}{n+k+1}\left(\begin{array}{c}
n+k+1 \\
2 k+1
\end{array}\right) 5^{k} F_{l}^{2 k} \\
& =F_{l} \sum_{k=0}^{n}(-1)^{l(n+k)} \frac{2 n+1}{2 k+1}\left(\begin{array}{c}
n+k \\
2 k
\end{array}\right) 5^{k} F_{l}^{2 k} .
\end{aligned}
$$

We have $F_{2 n}=\sum_{k=0}^{n-1}\left(\begin{array}{c}n+k \\ 2 k+1\end{array}\right), F_{4 n}=3 \sum_{k=0}^{n-1}(-1)^{(n+k+1)}\left(\begin{array}{c}n+k \\ 2 k+1\end{array}\right) 9^{k}, F_{6 n}=8 \sum_{k=0}^{n-1}\left(\begin{array}{c}n+k \\ 2 k+1\end{array}\right) 16^{k}, \ldots$

Dilcher said that the relation

$$
F_{4 n+2}=(2 n+1) \sum_{k=0}^{n} \frac{1}{2 k+1}\left(\begin{array}{c}
n+k \\
2 k
\end{array}\right) 5^{k}
$$

seem to be new (this is relation (5.7) in [5]). This is obtained by just putting $l=2$ in the last result of the previous theorem. These formulas were obtained by Jennings [9]; using a relatively long proof. Later, they were derived by Filipponi [7], using Waring' formula. By the same method, he also proved the following results:

Theorem 5.2. (Filipponi [7])

$$
\begin{aligned}
L_{2 n l} & =\sum_{k=0}^{n}(-1)^{l(n+k)} \frac{2 n}{n+k}\left(\begin{array}{c}
n+k \\
2 k
\end{array}\right) 5^{k} F_{l}^{2 k} \\
L_{2 n l} & =\sum_{k=0}^{n}(-1)^{(l+1)(n+k)} \frac{2 n}{n+k}\left(\begin{array}{c}
n+k \\
2 k
\end{array}\right) L_{l}^{2 k} \\
L_{(2 n+1) l} & =L_{l} \sum_{k=0}^{n}(-1)^{(l+1)(n+k)} \frac{2 n+1}{n+k+1}\left(\begin{array}{c}
n+k+1 \\
2 k+1
\end{array}\right) L_{l}^{2 k} \\
& =L_{l} \sum_{k=0}^{n}(-1)^{(l+1)(n+k)} \frac{2 n+1}{2 k+1}\left(\begin{array}{c}
n+k \\
2 k
\end{array}\right) L_{l}^{2 k} .
\end{aligned}
$$

By the formula

$$
X^{n}-Y^{n}=(X-Y) \sum_{k=0}^{\left\lfloor\frac{n-1}{2}\right\rfloor}(-1)^{k}\left(\begin{array}{c}
n-k-1 \\
k
\end{array}\right)(X Y)^{k}(X+Y)^{n-2 k+-1},
$$

(already used by Carlitz [3]), Swamy [14] established the following:

Theorem 5.3. (Swamy [14]) For positive integers $n, l \geq 1$ we have

$$
\begin{aligned}
F_{2 n l} & =F_{l} L_{l} \sum_{k=0}^{n-1}(-1)^{l(n+k+1)}\left(\begin{array}{c}
n+k \\
2 k+1
\end{array}\right) 5^{k} F_{l}^{2 k} \\
L_{(2 n+1) l} & =L_{l} \sum_{k=0}^{n}(-1)^{l(n+k)}\left(\begin{array}{c}
n+k \\
2 k
\end{array}\right) 5^{k} F_{l}^{2 k} .
\end{aligned}
$$


Note that in the first formula $l$ may be 0 . Dilcher [5] claimed that he was unable to find the formula $F_{4 n}=3 \sum_{k=0}^{n-1}\left(\begin{array}{c}n+k \\ 2 k+1\end{array}\right) 5^{k}$ in the literature. In fact this is known, it is obtained just by setting $l=2$ in the first relation of Swamy. In what follows, we will deduce all of these formulas, using just the Fibonacci polynomial $F_{n}(x)$, its anti-derivative and simple specializations for the unknown. Let us prove the previous results.

Proof. (Jennings results) For the first formula, let $x=i^{2 l+2} L_{l}^{2} \quad\left(i^{2}=-1\right)$ in $(2)$, By noting that

$$
\left(\frac{i^{l+1} L_{l}+\sqrt{4+i^{2 l+2} L_{l}^{2}}}{2}\right)^{2 n}=\alpha^{2 n l}
$$

and

$$
\left(\frac{i^{l+1} L_{l}-\sqrt{4+i^{2 l+2} L_{l}^{2}}}{2}\right)^{2 n}=\beta^{2 n l}
$$

we get

$$
\begin{aligned}
F_{2 n}(x) & =\sum_{k=0}^{n-1}\left(\begin{array}{c}
n+k \\
2 k+1
\end{array}\right)\left(i^{2 l+2} L_{l}^{2}\right)^{k} \\
\sum_{k=0}^{n-1}\left(\begin{array}{c}
n+k \\
2 k+1
\end{array}\right)(-1)^{k(l+1)} L_{l}^{2 k} & =\frac{(-1)^{l+1} i^{2(l+1) n}}{\sqrt{5} L_{l} F_{l}}\left(\left(\alpha^{l}\right)^{2 n}-\left(\beta^{l}\right)^{2 n}\right) \\
& =\frac{(-1)^{l+1+(l+2) n}}{\sqrt{5} L_{l} F_{l}}\left(\alpha^{2 n l}-\beta^{2 n l}\right) .
\end{aligned}
$$

Finally, we have the wanted result

$$
\sum_{k=0}^{n-1}\left(\begin{array}{c}
n+k \\
2 k+1
\end{array}\right)(-1)^{(n+k+1)(l+1)} L_{l}^{2 k}=\frac{1}{\sqrt{5} L_{l} F_{l}}\left(\alpha^{2 n l}-\beta^{2 n l}\right)=\frac{F_{2 n l}}{L_{l} F_{l}} .
$$

For the second, let $x=i^{2 l+2} L_{l}^{2}$ in (3). Using (4) we get

$$
\begin{aligned}
F_{2 n+1}\left(i^{2 l+2} L_{l}^{2}\right) & =\sum_{k=0}^{n}\left(\begin{array}{c}
n+k \\
2 k
\end{array}\right)\left(i^{2 l+2} L_{l}^{2}\right)^{k} \\
& =\frac{i^{(l+1)(2 n+1)}}{i^{l+1} \sqrt{5} F_{l}}\left(\left(\frac{L_{l}+\sqrt{5} F_{l}}{2}\right)^{2 n+1}-\left(\frac{L_{l}-\sqrt{5} F_{l}}{2}\right)^{2 n+1}\right)
\end{aligned}
$$

After simplification, we get

$$
\sum_{k=0}^{n}\left(\begin{array}{c}
n+k \\
2 k
\end{array}\right)(-1)^{(n+k)(l+1)}\left(L_{l}^{2}\right)^{k}=\frac{1}{\sqrt{5} F_{l}}\left(\alpha^{l(2 n+1)}-\beta^{l(2 n+1)}\right)=\frac{F_{(2 n+1) l}}{F_{l}} .
$$

For the last one, letting $x=i^{l} \sqrt{5} F_{l}$ in (7), and using (4), we get

$$
\left(\frac{i^{l} \sqrt{5} F_{l} \pm \sqrt{4+\left(i^{l} \sqrt{5} F_{l}\right)^{2}}}{2}\right)^{2 n+1}=i^{(2 n+1) l}\left(\frac{\sqrt{5} F_{l} \pm L_{l}}{2}\right)^{2 n+1}
$$




$$
\begin{aligned}
H_{2 n+1}(x) & =\sum_{k=0}^{n}\left(\begin{array}{c}
n+k \\
2 k
\end{array}\right) \frac{\left(i^{l} \sqrt{5} F_{l}\right)^{2 k+1}}{2 k+1} \\
& =\frac{i^{(2 n+1) l}}{2 n+1}\left(\left(\frac{\sqrt{5} F_{l}+L_{l}}{2}\right)^{2 n+1}+\left(\frac{\sqrt{5} F_{l}-L_{l}}{2}\right)^{2 n+1}\right) \\
& =\frac{i^{(2 n+1) l}}{2 n+1}\left(\left(\alpha^{l}\right)^{2 n+1}+\left(-\beta^{l}\right)^{2 n+1}\right) \\
& =\frac{i^{(2 n+1) l}}{2 n+1}\left(\alpha^{(2 n+1) l}-\beta^{(2 n+1) l}\right)
\end{aligned}
$$

So,

$$
\begin{aligned}
\sum_{k=0}^{n}\left(\begin{array}{c}
n+k \\
2 k
\end{array}\right) \frac{(-1)^{l(k+n)} 5^{k}\left(F_{l}\right)^{2 k}}{2 k+1} & =\frac{1}{(2 n+1) F_{l} \sqrt{5}}\left(\alpha^{l(2 n+1)}-\beta^{l(2 n+1)}\right) \\
& =\frac{F_{(2 n+1) l}}{(2 n+1) F_{l}},
\end{aligned}
$$

and the proof is finished.

Filipponi results are proved as follows:

Proof. (Filipponi results). First, note that for $n \geq 1$, we have

$$
L_{2 n}(x)=\sum_{k=0}^{n} \frac{2 n}{n+k}\left(\begin{array}{c}
n+k \\
2 k
\end{array}\right) x^{2 k}=\left(\frac{x+\sqrt{4+x^{2}}}{2}\right)^{2 n}+\left(\frac{x-\sqrt{4+x^{2}}}{2}\right)^{2 n} .
$$

Let $x=i^{l} \sqrt{5} F_{l}$ in (14), this yields

$$
\begin{aligned}
L_{2 n}\left(i^{l} \sqrt{5} F_{l}\right) & =\sum_{k=0}^{n} \frac{2 n}{n+k}\left(\begin{array}{c}
n+k \\
2 k
\end{array}\right)\left(i^{l} \sqrt{5} F_{l}\right)^{2} \\
& =\sum_{k=0}^{n} \frac{2 n}{n+k}\left(\begin{array}{c}
n+k \\
2 k
\end{array}\right)(-1)^{l k} 5^{k} F_{l}^{2 k} \\
& =(-1)^{2 n l}\left(\left(\frac{\sqrt{5} F_{l}+L_{l}}{2}\right)^{2 n}+\left(\frac{\sqrt{5} F_{l}-L_{l}}{2}\right)^{2 n}\right),
\end{aligned}
$$

We deduce

$$
\sum_{k=0}^{n} \frac{2 n}{n+k}\left(\begin{array}{c}
n+k \\
2 k
\end{array}\right)(-1)^{l(k+n)} 5^{k} F_{l}^{2 k}=\left(\alpha^{l}\right)^{2 n}+\left(\beta^{l}\right)^{2 n}=L_{2 n l} .
$$

For the second one, again let $x=i^{l} L_{l}$ in (14) :

$$
\begin{aligned}
L_{2 n}\left(i^{l} L_{l}\right) & =\sum_{k=0}^{n} \frac{2 n}{n+k}\left(\begin{array}{c}
n+k \\
2 k
\end{array}\right)\left(i^{l+1} L_{l}\right)^{2 k} \\
& =\sum_{k=0}^{n} \frac{2 n}{n+k}\left(\begin{array}{c}
n+k \\
2 k
\end{array}\right)(-1)^{(l+1) k} L_{l}^{2 k} \\
& =i^{2 n(l+1)}\left(\left(\frac{L_{l}+\sqrt{5} F_{l}}{2}\right)^{2 n}+\left(\frac{L_{l}-\sqrt{5} F_{l}}{2}\right)^{2 n}\right) .
\end{aligned}
$$


So,

$$
\sum_{k=0}^{n} \frac{2 n}{n+k}\left(\begin{array}{c}
n+k \\
2 k
\end{array}\right)(-1)^{(l+1)(k+n)} L_{l}^{2 k}=\alpha^{2 n l}+\left(-\beta^{l}\right)^{2 n}=L_{2 n l} .
$$

For the last relation of Filipponi, put $x=i^{l+1} L_{l}$ in (7). This yields

$$
H_{2 n+1}\left(i^{l+1} L_{l}\right)=\sum_{k=0}^{n}\left(\begin{array}{c}
n+k \\
2 k
\end{array}\right) \frac{\left(i^{l+1} L_{l}\right)^{2 k+1}}{2 k+1} .
$$

Since

$$
\left(\frac{i^{l+1} L_{l}+i^{l+1} \sqrt{5} F_{l}}{2}\right)^{2 n+1}=\alpha^{(2 n+1) l}
$$

and

$$
\begin{gathered}
\left(\frac{i^{l+1} L_{l}-i^{l+1} \sqrt{5} F_{l}}{2}\right)^{2 n+1}=\beta^{(2 n+1) l}, \\
\sum_{k=0}^{n}\left(\begin{array}{c}
n+k \\
2 k
\end{array}\right) \frac{i^{(l+1)(2 k+1)} L_{l}^{2 k+1}}{2 k+1}=\frac{i^{(l+1)(2 n+1)}}{2 n+1}\left(\left(\frac{L_{l}+\sqrt{5} F_{l}}{2}\right)^{2 n+1}+\left(\frac{L_{l}-\sqrt{5} F_{l}}{2}\right)^{2 n+1}\right) .
\end{gathered}
$$

Finally, we obtain

$$
\sum_{k=0}^{n}\left(\begin{array}{c}
n+k \\
2 k
\end{array}\right) \frac{(-1)^{(l+1)(n+k)} L_{l}^{2 k+1}}{2 k+1}=\frac{1}{2 n+1}\left(\alpha^{(2 n+1) l}+\beta^{(2 n+1) l}\right)=\frac{1}{2 n+1} L_{(2 n+1) l} .
$$

This finishes the proof of Filipponi results.

Now, let us prove the results due to Swamy.

Proof. (Swamy results)

Putting $x=\left(i^{l} \sqrt{5} F_{l}\right)^{2}$ in $(2)$ yields

$$
\begin{aligned}
F_{2 n}\left(\left(i^{l} \sqrt{5} F_{l}\right)^{2}\right) & =\sum_{k=0}^{n-1}\left(\begin{array}{c}
n+k \\
2 k+1
\end{array}\right)\left(i^{l} \sqrt{5} F_{l}\right)^{2 k} \\
& =\frac{1}{(i)^{2 l} \sqrt{5} F_{l} L_{l}}\left(A^{2 n}-B^{2 n}\right)
\end{aligned}
$$

where

$$
A=\left(\frac{i^{l} \sqrt{5} F_{l}+\sqrt{4+\left(i^{l} \sqrt{5} F_{l}\right)^{2}}}{2}\right)=\alpha^{l}
$$

and

$$
B=\left(\frac{i^{l} \sqrt{5} F_{l}-\sqrt{4+\left(i^{l} \sqrt{5} F_{l}\right)^{2}}}{2}\right)=\beta^{l} .
$$

Thus

$$
\sum_{k=0}^{n-1}(-1)^{l k}\left(\begin{array}{c}
n+k \\
2 k+1
\end{array}\right) 5^{k} F_{l}^{2 k}=\frac{(-1)^{l+n l}}{\sqrt{5} F_{l} L_{l}}\left(\left(\alpha^{l}\right)^{2 n}-\left(\beta^{l}\right)^{2 n}\right) .
$$


This is nothing than

$$
\sum_{k=0}^{n-1}(-1)^{l(n+k+1)}\left(\begin{array}{c}
n+k \\
2 k+1
\end{array}\right) 5^{k} F_{l}^{2 k}=\frac{F_{2 n l}}{F_{l} L_{l}}
$$

The second relation is obtained by letting $x=\left(i^{l} \sqrt{5} F_{l}\right)^{2}(l \geq 1)$ in $(3)$.

$$
\sum_{k=0}^{n}\left(\begin{array}{c}
n+k \\
2 k
\end{array}\right)(-1)^{l k} 5^{k} F_{l}^{2 k}=\frac{i^{l(2 n+1)}}{i^{l} L_{l}}\left(\left(\frac{\sqrt{5} F_{l}+L_{l}}{2}\right)^{2 n+1}-\left(\frac{\sqrt{5} F_{l}-L_{l}}{2}\right)^{2 n+1}\right),
$$

After simplification we get,

$$
\sum_{k=0}^{n}\left(\begin{array}{c}
n+k \\
2 k
\end{array}\right)(-1)^{l(k+n)} 5^{k} F_{l}^{2 k}=\frac{1}{L_{l}}\left(\left(\alpha^{l}\right)^{2 n+1}+\left(\beta^{l}\right)^{2 n+1}\right)=\frac{L_{l(2 n+1)}}{L_{l}} .
$$

This is the wanted result,

The polynomials $F_{2 n}(x)$ and $F_{2 n+1}(x)$ may be used to find other identities. For instance, using the hypergeometric representation of the Fibonacci number, Dilcher [5] gave the formulas

$$
F_{2 n}=\frac{1}{2} \sum_{k=0}^{2 n-1}(-1)^{k+1}\left(\begin{array}{c}
2 n+k \\
2 k+1
\end{array}\right) F_{3 k}
$$

and

$$
F_{2 n+1}=(2 n+1) \sum_{k=0}^{2 n-1}(-1)^{k+1}\left(\begin{array}{c}
2 n+k+1 \\
2 k
\end{array}\right) \frac{F_{3 k}}{2 n+k+1} .
$$

Let us prove the first one using just $F_{n}(x)$

Proposition 5.2. For $n \geq 1$,

$$
F_{2 n}=\frac{1}{2} \sum_{k=0}^{2 n-1}(-1)^{k+1}\left(\begin{array}{c}
2 n+k \\
2 k+1
\end{array}\right) F_{3 k} .
$$

Proof. Consider

$$
\begin{aligned}
F_{4 n}(x) & =\sum_{k=0}^{2 n-1}\left(\begin{array}{l}
2 n+k \\
2 k+1
\end{array}\right) x^{k} \\
& =\frac{1}{\sqrt{x(x+4)}}\left(\left(\frac{\sqrt{x}+\sqrt{4+x}}{2}\right)^{4 n}-\left(\frac{\sqrt{x}-\sqrt{4+x}}{2}\right)^{4 n}\right) \\
& =\frac{1}{\sqrt{x(x+4)}}\left(\left(\frac{x+2+\sqrt{x(4+x)}}{2}\right)^{2 n}-\left(\frac{x+2-\sqrt{x(4+x)}}{2}\right)^{2 n}\right) .
\end{aligned}
$$

Let $x=-(2 \pm \sqrt{5})=-\left(\frac{1 \pm \sqrt{5}}{2}\right)^{3}$ in the previous formula, we get

$$
\begin{aligned}
F_{4 n}(-(2 \pm \sqrt{5})) & =\sum_{k=0}^{2 n-1}(-1)^{k}\left(\begin{array}{c}
2 n+k \\
2 k+1
\end{array}\right)(2 \pm \sqrt{5})^{k} \\
& =\left(\left(\frac{1 \mp \sqrt{5}}{2}\right)^{2 n}-\left(\frac{1 \pm \sqrt{5}}{2}\right)^{2 n}\right) .
\end{aligned}
$$


Now just substract to get

$$
\sum_{k=0}^{2 n-1}(-1)^{k}\left(\begin{array}{l}
2 n+k \\
2 k+1
\end{array}\right)\left((2+\sqrt{5})^{k}-(2-\sqrt{5})^{k}\right)=2\left(\left(\frac{1-\sqrt{5}}{2}\right)^{2 n}-\left(\frac{1+\sqrt{5}}{2}\right)^{2 n}\right) .
$$

So, after multiplying by $-1 / 2 \sqrt{5}$, we get

$$
F_{2 n}=\frac{1}{2} \sum_{k=0}^{2 n-1}(-1)^{k+1}\left(\begin{array}{c}
2 n+k \\
2 k+1
\end{array}\right) F_{3 k} .
$$

The other identity involving $F_{2 n+1}$ may be proved similarly.

\section{Identities involving the derivatives}

In what follows, we give some (known) identities involving the derivatives of Fibonacci polynomials. Melham [12] proved, among others, the following identities:

Theorem 6.1. (Melham)

$$
\begin{aligned}
\frac{F_{(2 n+1) l}}{F_{l}} & =\sum_{r=0}^{n}(-1)^{n+l(n+r)} 2^{-r} L_{2 l}^{r} \sum_{k=r}^{n}(-2)^{k}\left(\begin{array}{c}
k \\
r
\end{array}\right)\left(\begin{array}{c}
n+k \\
2 k
\end{array}\right) \\
\frac{L_{(2 n+1) l}}{L_{l}} & =\sum_{r=0}^{n}(-1)^{r+l(n+r)} 2^{-r} L_{2 l}^{r} \sum_{k=r}^{n}(-2)^{k}\left(\begin{array}{c}
k \\
r
\end{array}\right)\left(\begin{array}{c}
n+k \\
2 k
\end{array}\right)
\end{aligned}
$$

He also evaluated several sums of the following kind

Lemma 6.1. (Melham) For $n \geq 1$ and $r \geq 0$

$$
\begin{aligned}
& \sum_{k=r}^{n}(-2)^{k}\left(\begin{array}{l}
k \\
r
\end{array}\right)\left(\begin{array}{c}
n+k \\
2 k
\end{array}\right)=\left\{\begin{array}{cc}
(-1)^{\frac{n+r}{2}} 2^{r}\left(\begin{array}{c}
\frac{n+r}{2} \\
r
\end{array}\right), n \equiv r \quad \bmod (2) \\
(-1)^{\frac{n+r+1}{2}} 2^{r}\left(\begin{array}{c}
\frac{n+r-1}{2} \\
r
\end{array}\right), n \equiv r+1 \quad \bmod (2)
\end{array}\right. \\
& \sum_{k=r}^{n-1}(-2)^{k}\left(\begin{array}{c}
k \\
r
\end{array}\right)\left(\begin{array}{c}
n+k \\
2 k+1
\end{array}\right)=\left\{\begin{array}{ccc}
0, & n \equiv r & \bmod (2) \\
(-1)^{\frac{n+r-1}{2}} 2^{r}\left(\begin{array}{c}
\frac{n+r-1}{2} \\
r
\end{array}\right), & n \equiv r+1 & \bmod (2)
\end{array}\right.
\end{aligned}
$$

Using just the polynomial $F_{2 n}(x)$ and its derivatives, Melham results may be stated as follows:

\section{Theorem 6.2.}

$$
\begin{aligned}
\frac{F_{(2 n+1) l}}{F_{l}} & =\sum_{r=0}^{n}(-1)^{(l+1)(n+r)} L_{2 l}^{r} \frac{F_{2 n+1}^{(r)}(-2)}{r !} \\
\frac{L_{(2 n+1) l}}{L_{l}} & =\sum_{r=0}^{n}(-1)^{(n+r)} L_{2 l}^{r} \frac{F_{2 n}^{(r)}(-2)}{r !} .
\end{aligned}
$$


Proof. Note that

$$
\begin{aligned}
\sum_{k=r}^{n}(-2)^{k}\left(\begin{array}{l}
k \\
r
\end{array}\right)\left(\begin{array}{c}
n+k \\
2 k
\end{array}\right) & =\sum_{k=r}^{n}(-2)^{k} \frac{k(k-1)(k-2) \ldots(k-r+1)}{r !}\left(\begin{array}{c}
n+k \\
2 k
\end{array}\right) \\
& =\frac{(-2)^{r}}{r !} F_{2 n+1}^{(r)}(-2) .
\end{aligned}
$$

Replace this in the previous theorem to get the identities of this theorem. Hence, again these identities may be expressed by Fibonacci polynomial and its derivatives.

\section{Conclusion}

Integrating (10) and (11), $j$ times we obtain the following polynomials:

$$
\begin{gathered}
\sum_{k=0}^{\left\lfloor\frac{n}{2}\right\rfloor}\left(\begin{array}{c}
n+j \\
2 k+j
\end{array}\right) x^{2 k+j}=\frac{1}{2}\left((1+x)^{n+j}+(-1)^{j}(1-x)^{n+j}\right) \\
\sum_{k=0}^{\left\lfloor\frac{n-1}{2}\right\rfloor}\left(\begin{array}{c}
n+j \\
2 k+j+1
\end{array}\right) x^{2 k+j+1}=\frac{1}{2}\left((1+x)^{n+j}+(-1)^{j-1}(1-x)^{n+j}\right) .
\end{gathered}
$$

From these, it is possible to deduce many identities, for example, letting $x=\frac{\sqrt{5} F_{l}}{L_{l}}$ in the previous polynomial, and replace $j$ by $2 j$, we get

$$
2^{1-n-2 j} \sum_{k=0}^{\left\lfloor\frac{n-1}{2}\right\rfloor}\left(\begin{array}{c}
n+2 j \\
2 k+2 j+1
\end{array}\right)\left(5 F_{l}^{2}\right)^{k+1} L_{l}^{n-2 k}=\frac{L_{l}}{F_{l}} F_{l(n+2 j)} .
$$

Put $x^{2}$ instead of $x$, we get

$$
\begin{gathered}
\sum_{k=0}^{\left\lfloor\frac{n-1}{2}\right\rfloor}\left(\begin{array}{c}
n \\
2 k
\end{array}\right) x^{4 k+2}=\frac{1}{2}\left(\left(1+x^{2}\right)^{n}-\left(1-x^{2}\right)^{n}\right) \\
\sum_{k=0}^{\left\lfloor\frac{n-1}{2}\right\rfloor}\left(\begin{array}{c}
n \\
2 k+1
\end{array}\right) x^{4 k}=\frac{1}{2}\left(\left(1+x^{2}\right)^{n}+\left(1-x^{2}\right)^{n}\right),
\end{gathered}
$$

their antiderivatives involve the hyperbolic functions. It is possible to use these polynomials and their derivatives to find new proofs, or even (may be) new identities for Fibonacci and Lucas numbers.

Pell and Pell-Lucas sequences are defined by

$$
\begin{aligned}
& P_{1}=, P_{2}=2, \text { and for } n \geq 2, P_{n}=2 P_{n-1}+P_{n-2} \\
& Q_{1}=1, Q_{2}=3, \text { and for } n \geq 2, Q_{n}=2 Q_{n-1}+Q_{n-2}
\end{aligned}
$$


Their generating polynomials are defined by

$$
\begin{aligned}
P_{n}(x) & =\sum_{k=0}^{\left\lfloor\frac{n-1}{2}\right\rfloor}\left(\begin{array}{c}
n-k-1 \\
k
\end{array}\right)(2 x)^{n-2 k-1}=\frac{1}{2 \sqrt{x^{2}+1}}\left(\left(x+\sqrt{x^{2}+1}\right)^{n}-\left(x-\sqrt{x^{2}+1}\right)^{n}\right) \\
Q_{n}(x) & =\sum_{k=0}^{\left\lfloor\frac{n}{2}\right\rfloor}\left(\begin{array}{c}
n-k \\
k
\end{array}\right)(2 x)^{n-2 k}=\left(x+\sqrt{x^{2}+1}\right)^{n}+\left(x-\sqrt{x^{2}+1}\right)^{n} .
\end{aligned}
$$

Pell polynomials are related to the Fibonacci's by the relation $P_{n}(x / 2)=\phi_{n}(x)$. So, it is possible to use the same elementary techniques to derive other proofs and may be new identities concerning these numbers.

Finally we ask if there is a general formula (like Seiffert [13]) for the Lucas polynomials. Another question is: does every formula involving Fibonacci and Lucas numbers (any formula with finite sum) may be proved using the Fibonacci polynomial and its relatives?

\section{Acknowledgements}

The author would to thank the anonymous referees for their corrections and suggestions which improved the presentation of the paper. The thanks are also due to the NNTDM Technical Editor, for her helpful remarks.

\section{References}

[1] Ait-Amrane, L. (2019). Larger families of solutions to some Diophantine equations. Notes on Number Theory and Discrete Mathematics, 251, 25-31.

[2] Brown, J., Dilcher, K., Manna, D. (2012). Series representations of theta functions in terms of a sequence of polynomials. The Fibonacci Quarterly, 50(1), 5-10.

[3] Carlitz, L. (1975). Some identities of Bruckman. The Fibonacci Quarterly, 13(2), 121-126.

[4] Carlitz, L. (1963). A Fibonacci Array. The Fibonacci Quarterly, 1(2), 17-28.

[5] Dilcher, K. (2000). Hypergeometric functions and Fibonacci numbers. The Fibonacci Quarterly, 38(4), 342-363.

[6] Filipponi, P., \& Horadam, A. (1993). Second derivative sequences of Fibonacci and Lucas polynomials. The Fibonacci Quarterly, 31(3), 194-204.

[7] Filipponi, P. (1995). Some binomial Fibonacci identities. The Fibonacci Quarterly, 33(3), 251-257.

[8] Gould, H. W. (1977). A Fibonacci formula of Lucas and its subsequent manifestations and rediscoveries. The Fibonacci Quarterly, 15(1), 25-29. 
[9] Jennings, D. (1993). Some polynomial identities for the Fibonacci and Lucas numbers. The Fibonacci Quarterly, 31(2), 134-137.

[10] Keskin, R. (2014). Three identities concerning Fibonacci and Lucas numbers. Notes on Number Theory and Discrete. Vol. 20, No. 5, (2014) 44-48.

[11] Lucas, E. (1969). The Theory of Simply Periodic Numerical Functions. The Fibonacci Association. Translated by Sidney Kravitz and Edited by Douglas Lind. (1969)

[12] Melham, R. S. (1999). On certain polynomials of even subscripted Lucas numbers. Applications of Fibonacci numbers, Ed. Fredric T. Howard, Vol. 8, 251-258.

[13] Seiffert, H.-J. (1997). Solution to problem H-508. The Fibonacci Quarterly, 35(2), 188-190.

[14] Swamy, M. N. S. (1997). Some identities in Fibonacci polynomials. The Fibonacci Quarterly, 35(3), 230-232. 